\title{
QUANTUM MODELLING OF POLY(VINYLIDENE FLUORIDE)
}

\author{
Helena M. G. Correia, Marta M. D. Ramos* \\ Departamento de Física, Universidade do Minho, Campus de Gualtar, \\ 4710-057 Braga, Portugal
}

\begin{abstract}
Although extensive studies have been conducted on poly(vinylidene fluoride) (PVDF) because of its ferroelectric, pyroelectric and piezoelectric properties, the effects of inverted monomer units, on the molecular scale properties of this polymer are not fully understood. Therefore, we have used a method combining molecular dynamics with a self-consistent semi-empirical quantum mechanical method to study the effects of both chain length and monomer inversion on the electronic properties of individual PVDF chains, such as the dipole moment and the polarizability. The effects of monomer inversion on the infrared spectra are also discussed.

Our results suggest that alpha and beta polymorphs of PVDF have dipole moment per monomer unit that varies (increases for beta-PVDF and decreases for alpha-PVDF) with the chain length but converges to a nearly constant value for chain lengths greater than a certain value, whereas chain length does not seem to produce any significant effect on molecular polarizability of both polymorphs. Our calculations also suggest a decrease of the dipole moment with increasing inversion monomer defect concentration but no significant effect has been predicted for molecular polarizability, except when two or more isolated defects are present in the same chain. The presence of monomer inverted defects decreases the intensity of the infrared peaks attributable to defect-free chains and gives rise to new peaks. The number and frequency of the defect peaks depends both on the isolated defect concentration and molecular chain structure.
\end{abstract}

Keywords: Quantum modelling, monomer inversion, dipole moment, polarizability, PVDF.

* Corresponding Author: Tel:+351 253 604330; Fax:+351 253678 981; E-mail: marta@ fisica.uminho.pt 


\section{Introduction}

Since the discovery of piezoelectricity in poly(vinylidene fluoride) (PVDF), $\left[\mathrm{H}-\left(\mathrm{CH}_{2}-\mathrm{CF}_{2}\right)_{\mathrm{n}}-\mathrm{H}\right]$, first reported by Kawai in 1967 [1], this polymer has attracted a great deal of attention as a material for piezoelectric applications $[2,3]$. However, inverted monomer units $\left[{ }^{\cdots} \mathrm{CH}_{2}-\mathrm{CF}_{2}-\boldsymbol{C F}_{2}-\boldsymbol{C H}_{2}-\right.$ $\left.\mathrm{CH}_{2}-\mathrm{CF}_{2} \cdots\right]$ arising during the polymerization process in PVDF have not been properly understood. An understanding of the possible structure-to-property relations is therefore of great importance. This provided the motivation for the work reported in this paper.

The inversion defect formation is directly influenced by the way in which the polymerization has taken place. Therefore, different polymerization techniques can lead to different inversion defect concentration [2]. A better knowledge of the effects of varying the number of inverted monomer units and their localization on the permanent electric molecular dipole moment and molecular polarizability has the potential to yield significant progress for designing polymer materials with enhanced piezoelectric activity.

The aim of this work is to perform atomistic modelling of chain growth in the polymerization of PVDF, with and without monomer inversion defects, to shed some light on the relationship between molecular structure and the electrical properties such as permanent electric molecular dipole moment and molecular polarizability. The effects of defect concentration and aggregation on the infrared spectra of PVDF are also discussed.

\section{Theoretical method}

In the present work we use the CHEMOS code $[4,5]$ that combines a self-consistent quantum mechanical method, working at CNDO (Complete Neglect of Differential Overlap) level of theory, with a molecular dynamics method which uses the atomic forces calculated self-consistently at each 
time step without numerical differentiation. This code has been used to calculate the optimised geometry of PVDF chains with different lengths, to obtain the Cartesian components of their molecular dipole moment $\left(\mu_{x}, \mu_{y}, \mu_{z}\right)$, by using the self-consistent-field wave functions, as well as the molecular polarizability, $\alpha_{x y}^{m o l}$, defined as the linear response to an external applied electrical field [6]:

$$
\mu_{x}^{\text {ind }}=\alpha_{x y}^{\text {mol }} E_{y}^{e x t}
$$

where $\mu_{x}^{\text {ind }}$ is the induced molecular dipole moment, $E_{y}^{e x t}$ is the external applied field and $x, y, z$ represent the Cartesian components.

In the calculations described above we proceed as follows: (i) select a starting geometry for the polymer chain; (ii) perform electronic structure calculations using the semi-empirical application of the Hartree-Fock theory called CNDO until self-consistency in energy is achieved; (iii) evaluate the energy gradient and calculate the forces acting on all atoms; (iv) move the atoms according to Newton's equations of motion during a time-step of 1fs; (v) repeat steps (ii) - (iv) until atomic relaxation is achieved. The CNDO calculations were performed using a set of parameters to give the best overall fit to the minimum basis set $a b$ initio Hartree-Fock calculations, a linear combination of atomic orbitals and a cluster model framework.

Once we have the optimised geometry of PVDF chains, with and without inversion monomer defects, we use the commercial software HyperChem to perform a vibrational analysis at this geometry and to calculate the infrared vibrational spectra using the CNDO method.

The vibrational spectra result from the interactions of the vibrational motions of the polymer chain with infrared electromagnetic radiation. These vibrational interactions are described by a simple harmonic oscilator model. HyperChem models the vibrations of the polymer chain as a set of point masses (the nuclei of the atoms) with each vibrating about its equilibrium (optimised) position. 
Although the energies and forces are evaluated quantum mechanically by the CNDO method, the vibration frequencies are derived from the harmonic approximation using Newton's equation of motion.

\section{Results and discussion}

\subsection{The effects of chain length}

A typical polymer consists of molecular chains with some statistical distribution of lengths. In order to study the effect of chain length on the electrical properties of both alpha and beta-PVDF, we have calculated the norm of the permanent dipole moment vector, $\mu=\left(\mu_{x}^{2}+\mu_{y}^{2}+\mu_{z}^{2}\right)^{1 / 2}$, and the mean polarizability, $\alpha=\frac{1}{3}\left(\alpha_{x x}+\alpha_{y y}+\alpha_{z z}\right)[6]$, as a function of chain length. Results of these calculations, shown in figures 1 and 2, have two main features. First, the dipole moment decreases as chain length increases for alpha-PVDF, whereas beta-PVDF shows an opposite behaviour. For both polymorphs the dipole moment per monomer unit converges to a nearly constant $\left(5 \times 10^{-30}\right.$ C.m for alpha-PVDF and $8 \times 10^{-30} \mathrm{C} . \mathrm{m}$ for beta-PVDF) value for a chain length greater than a certain number of monomer units (12 for alpha-PVDF and 17 for beta-PVDF. The magnitudes of the calculated dipole moment per monomer unit for a chain with 20 monomer units $\left(5.2 \times 10^{-30} \mathrm{C} . \mathrm{m}\right.$ for alpha-PVDF and $8.3 \times 10^{-30}$ C.m for beta-PVDF) are different from the value calculated from C-F and C-H bonds (7.0x10 $10^{-30}$ C.m) [7]. Secondly, chain length does not seem to produce a significant effect on polarizability for both alpha- and beta-PVDF.

The dipole moment calculated by the CNDO method is the sum of two terms. One term arises from the contribution of atomic charges and the other results from the displacement of charge density on the atoms due to mixing of atomic orbitals. The relative contribution of both terms to the dipole moment depends on chain geometry. As a result, all cartesian components of alpha-PVDF dipole 
moment do not change with length for chains with a complete trans-gauche-trans-gauche conformation (chains with a number of monomer units greater than five), whereas for a beta-PVDF with all-trans conformation only the dominant component of the dipole moment parallel to $\mathrm{CF}_{2}$ and $\mathrm{CH}_{2}$ dipoles increases with chain length and the other components change in the opposite way with chain length.

\subsection{The effects of inversion monomer defects}

The effects of inversion monomer defects in both alpha- and beta-PVDF were found by examining a single molecule of PVDF with 20 monomer units. The calculated optimized structures of alphaand beta-PVDF with and without inverted monomer units are shown in figure 3 . The consequences of the addition of 1 to 3 inverted monomer units in alpha-PVDF and beta-PVDF at different chain positions are shown in figure 4, 5 and 6. The dipole moment decreases with defect concentration (see figures 4 and 6), the effect being stronger for beta-PVDF, but does not seem to be affected by defect localization in the chain (figure 4). The presence of inverted monomer units in both alpha and beta-PVDF chains increases their mean polarizability less than $1 \%$ (figure 5). The mean

polarizability remains roughly constant when the inverted monomer defects are bound but increases slightly for isolated defects. In fact, bounded defects behave like a single defect of larger length.

Figures 7 and 8 show the infrared spectra of alpha-PVDF without defects and with two isolated inverted monomer defects, respectively. The introduction of defects shows three main features. First, there is a decrease in the intensity of defect-free peaks. Second, the inverted monomer defects give rise to new peaks with wave number in the regions of $830-850 \mathrm{~cm}^{-1}$ and $4550-4620 \mathrm{~cm}^{-1}$. Finally, there is a splitting of the peaks associated with the inverted monomer defects. The number of spectral multiplets predicted is equal to the number of isolated defects present in the PVDF chain. The calculated wave numbers of the defect multiplets are substantially different from the infrared 
bands at $1450,1320-1340$ and $678 \mathrm{~cm}^{-1}$ of commercial PVDF samples assigned to the localized modes associated with inversion monomer defects involved in the samples [8].

In beta-PVDF the presence of inverted monomer defects is predicted to give rise to a single defect peak with wave number only in region of $820-845 \mathrm{~cm}^{-1}$, which is splitted in a number of spectral multiplets similar to what was predicted for alpha-PVDF. The wave number of the defect multiplet computed by CNDO method is higher than the corresponding experimental value $\left(677 \mathrm{~cm}^{-1}\right)$ [9].

Comparison between vibrational frequencies calculated using CNDO method and their experimental values confirms the observation of an apparently over-prediction of CNDO [4, 10] values. This is in agreement with the well-known result that CNDO over predicts force constants by a factor typically between 2 and $3[11,12]$. Despite the fact that the absolute value of calculated infrared vibrational frequencies may not be correct, we expect the predicted trends for alpha and beta-PVDF to be reliable.

\section{Conclusions}

We have studied the relationship between the molecular structure and the properties of PVDF polymorphs. Our results suggest the dipolar moment and molecular polarizability per monomer unit of both alpha and beta polymorphs change with chain length in opposite ways, the effects being stronger for the dipole moment. Therefore, the constant value of the dipole moment per monomer unit used in the interpretation of the PVDF experiments is in considerable error specially for short chains. For polymer chains with inverted monomer defects, the calculations predict a decrease in the dipole moment as the defect concentration increases, the effect being stronger for beta-PVDF. Moreover, these defects induce new infrared vibrational peaks which are splitted when there are two or more isolated defects in the PVDF chain. Therefore, one needs to be cautious in the 
interpretation of infrared spectra since the correlation of defect peaks with defect concentration is not straightforward.

The quantum molecular dynamics method used in this paper seems to be a valuable approach, because it permits prediction of general trends even though quantitative agreement with experimental data is often lacking. Improvements can be achieved by moving from standard CNDO parameters to ones determined by calibration with an experimental data set, rather than an overall fit to minimal basis set "ab initio" calculations. Our quantum modelling can also be combined with a mesoscopic modelling to elucidate many unanswered questions raised by piezoelectric experiments, such as the physical mechanisms involved in mechanical stretching in order to transform the alpha-PVDF, formed during simple crystallization upon cooling, into the beta-phase as well as in the poling (effect of an applying electric field) in order to optimise the piezoelectric properties of PVDF.

\section{Acknowledgement}

This work is part of the research project POCTI/CTM/41574/2001, approved by Portuguese Foundation for Science and Technology (FCT) and POCTI and supported by the European Community Fund FEDER. One of us (H.M.G.C.) is also indebted to FCT for financial support under PhD grant No. SFRH/BD/11231/2002.

\section{References}

[1] H. Kawai, Jpn. J. Appl. Phys., 8 (1969) 1975.

[2] A. J. Lovinger, in I.D.C. Basset (Eds), Developments in Crystalline Polymers, Applied Science, London, 1982.

[3] H. S. Nalwa, J. Macromol. Sci. C 31 (1991) 341.

[4] D. Wallace, D. Phil. Thesis, University of Oxford (1989). 
[5] D. S. Wallace, A. M. Stoneham, W. Hayes, A. J. Fisher, A. H. Harker, J. Phys. Condens. Matter, 3 (1991) 3879.

[6] L. Solymar and D. Walsh, "Lectures on the Electrical Properties of Materials", $4^{\text {th }}$ Edition, Oxford University Press, Oxford, 1989.

[7] R. G. Kepler, Ann. Rev. Phys. Chem. 20 (1978) 497.

[8] M. Kobayashi, K. Tashiro and H. Tado koro, Macromolecules, 8 (1975) 158.

[9] S. Lanceros-Méndez, J. F. Mano, A. M. Costa and V. H. Schmidt, J. Macrom. Sci. Phys., B, 40 (2001) 517.

[10] D. M. Seeger, C. Korzenienwski and W. Kowalchyl, J. Phys. Chem., 95 (1991) 68.

[11] J. Sadley, "Semi-empirical Methods in Quantum Chemistry", Ellis Horwood, Chischester, 1985.

[12] J. A. Pople and D. L. Beveridge, "Approximate Molecular Orbital Theory", McGraw-Hill, New York, 1970. 


\section{List of figure captions}

Figure 1 - Variation of the dipole moment per monomer unit of poly(vinylidene fluoride) (PVDF) $\left[\mathrm{H}-\left(\mathrm{CH}_{2}-\mathrm{CF}_{2}\right)_{\mathrm{n}}-\mathrm{H}\right]$ as a function of the chain length. The open and closed marks indicate the data points that were calculated explicitly for beta and alpha-PVDF, respectively.

Figure 2 - Variation of the mean polarizability per monomer unit of on poly(vinylidene fluoride) (PVDF) $\left[\mathrm{H}-\left(\mathrm{CH}_{2}-\mathrm{CF}_{2}\right)_{n}-\mathrm{H}\right]$ as a function of the chain length. The open and the closed marks indicate the data points that were calculated explicitly for beta and alpha-PVDF, respectively.

Figure 3 - View of the $-\left(\mathrm{CH}_{2}-\mathrm{CF}_{2}\right)-$ monomer and the optimised molecular structure of: a) defect-free alpha-PVDF chain; b) alpha-PVDF chain containing two isolated inversion monomer defects; c) defect-free beta-PVDF chain and d) beta-PVDF chain containing two isolated inversion monomer defects. The arrows indicate the position of the inversion monomer defects.

Figure 4 - Dependence of the dipole moment of alpha and beta-PVDF chains with 20 monomer units, shown in figure $3 \mathrm{a}$ and $3 \mathrm{c}$, on the number of inverted monomer units for defects localized at chain end (e), middle of the chain (m) and isolated (i). The lines are a guide to the eye.

Figure 5 - Dependence of the mean polarizability of alpha and beta-PVDF chains with 20 monomer units, shown in figure $3 \mathrm{a}$ and $3 \mathrm{c}$, on the number of inverted monomer units for defects localized at chain end (e), middle of the chain (m) and isolated (i). The lines are a guide to the eye.

Figure 6 -The effect of inversion defect concentration on the decrease of dipole moment of both alpha and beta-PVDF chains with 20 monomer units. 
Figure 7 - Simulated infrared vibrational spectrum of defect-free alpha-PVDF chain with 20 monomer units.

Figure 8 - The effect of two isolated inversion monomer defects on the infrared vibrational spectrum of alpha-PVDF chain with 20 monomer units. 
Figure 1

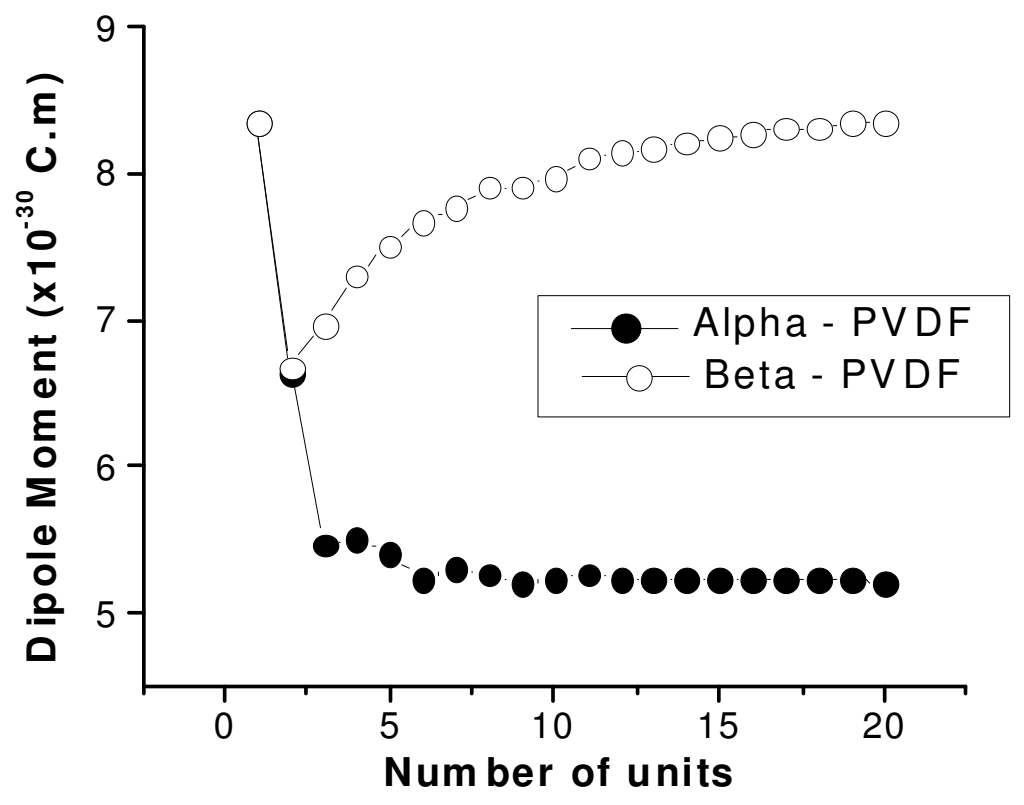


Figure 2

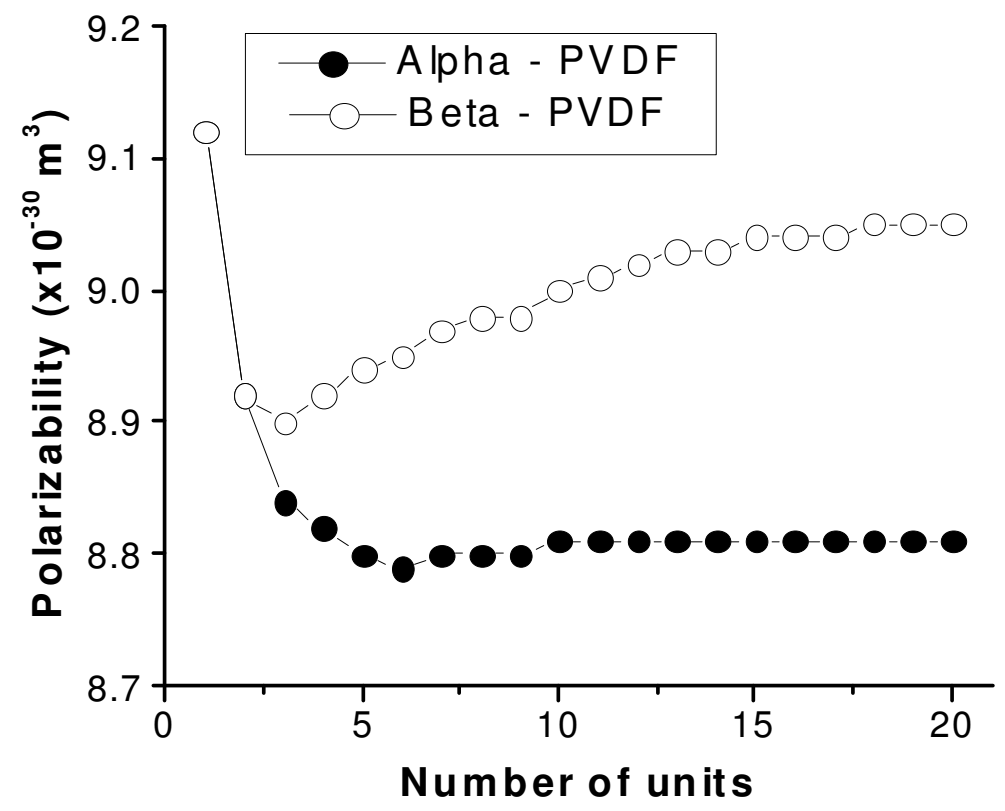


Figure 3

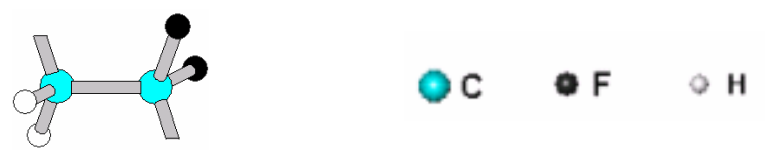

(a)

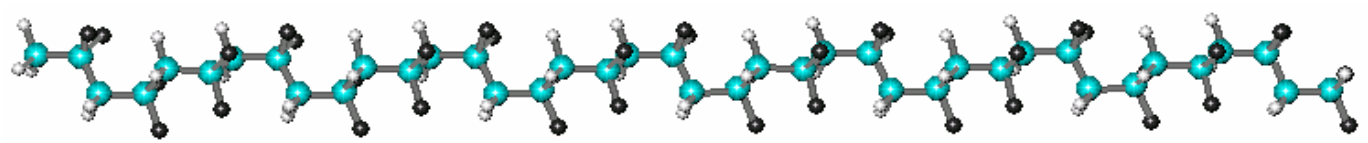

(b)

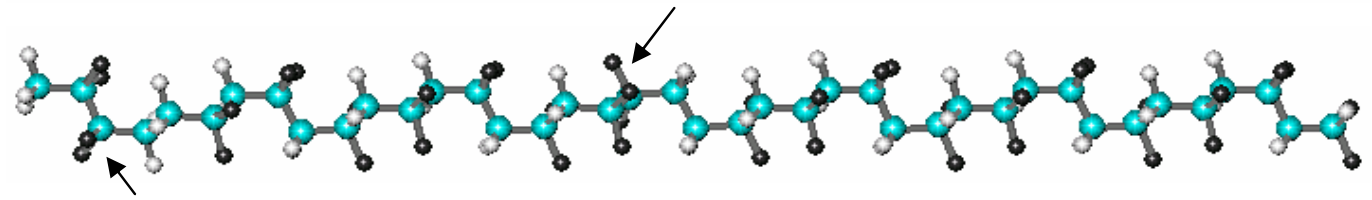

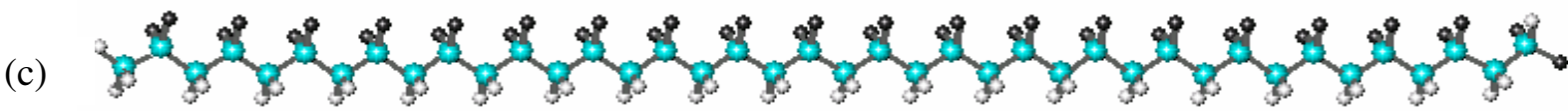

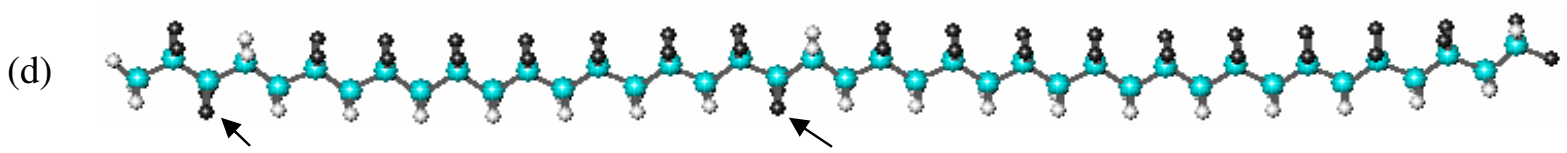


Figure 4

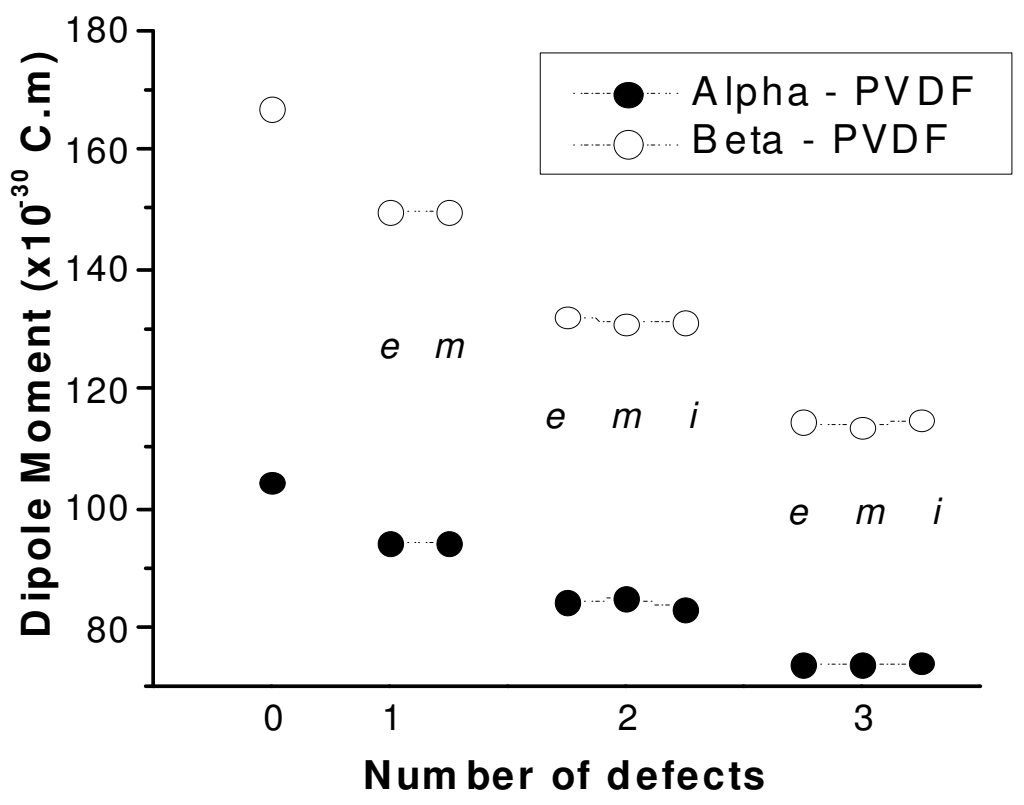


Figure 5

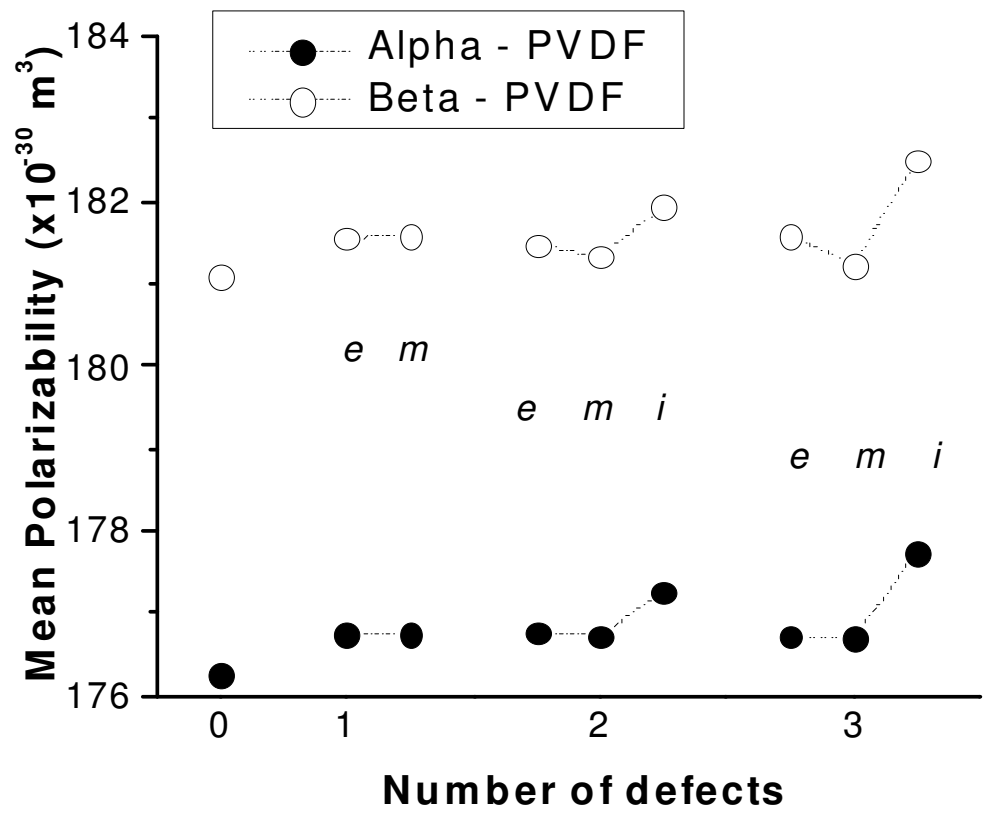


Figure 6

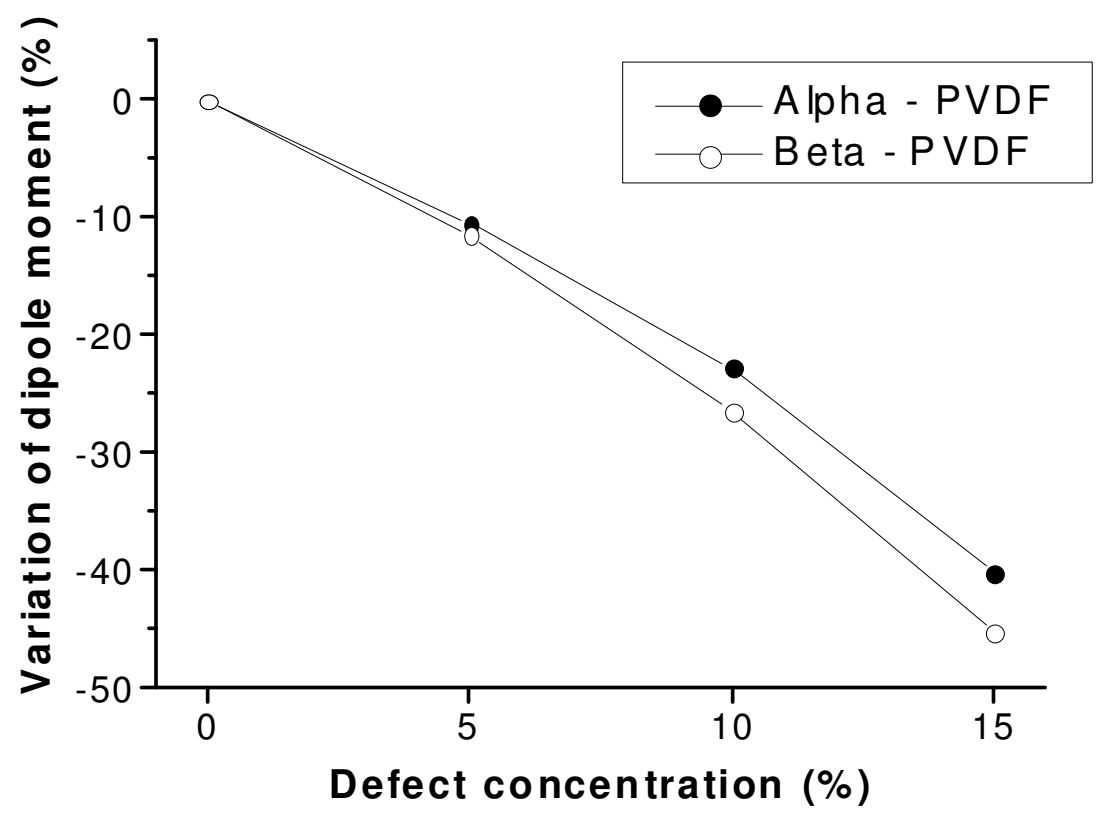


Figure 7

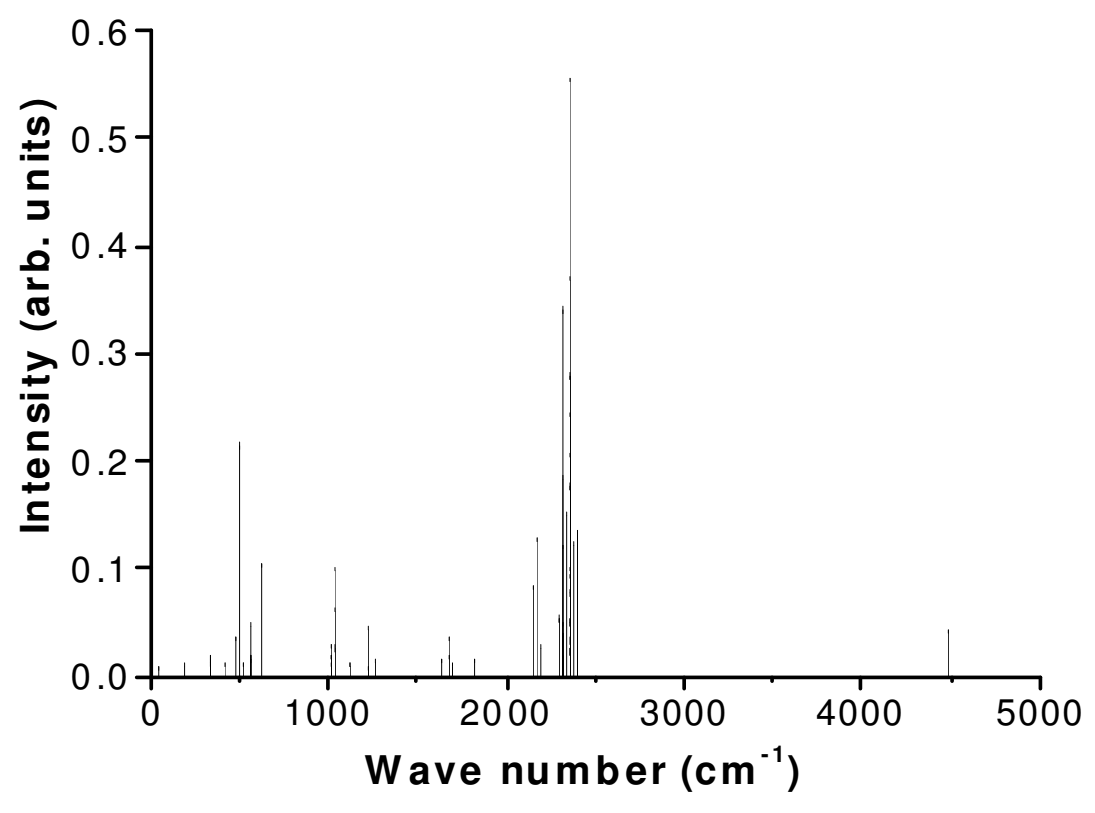


Figure 8

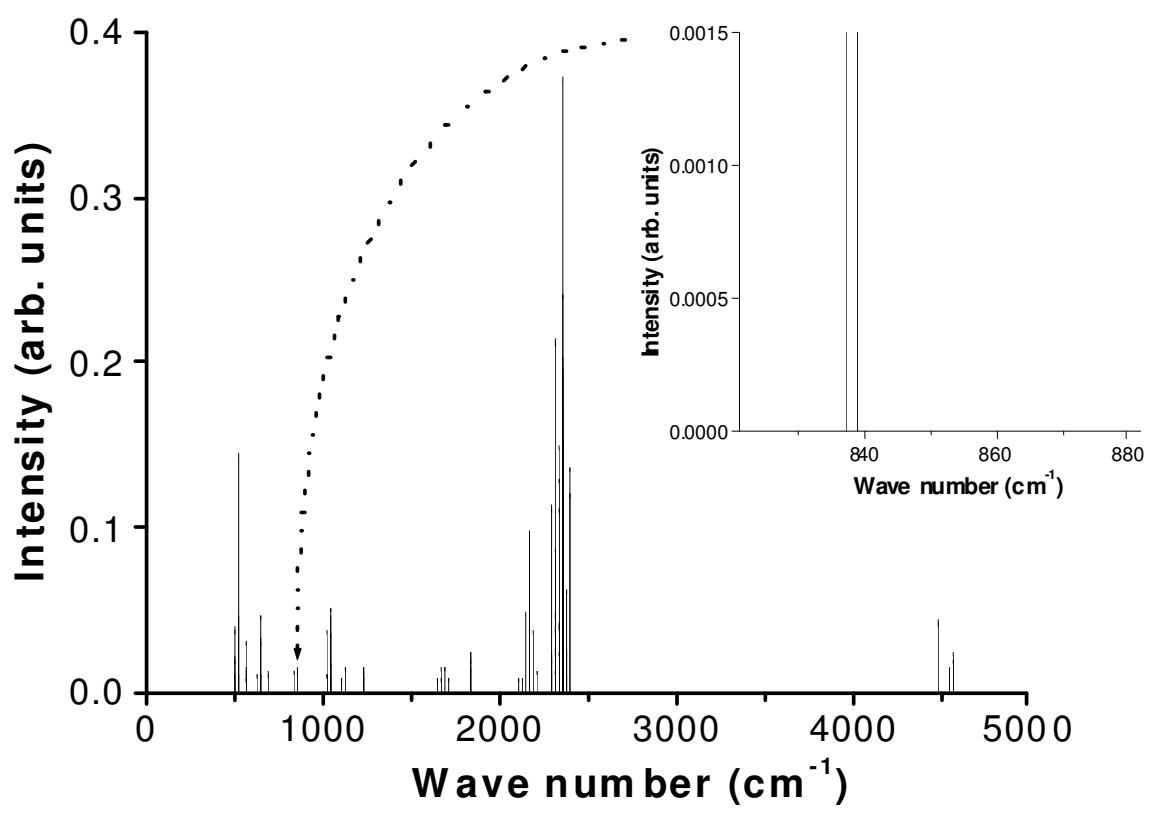

